EPJ Web of Conferences 107, 04001 (2016)

DOI: $10.1051 /$ epjconf/201610704001

(C) Owned by the authors, published by EDP Sciences, 2016

\title{
Pairing properties of realistic effective interactions
}

\author{
A. Gargano ${ }^{1, a}$, L. Coraggio ${ }^{1}$, A. Covello ${ }^{2}$, and N. Itaco ${ }^{1,2}$ \\ ${ }^{1}$ Istituto Nazionale di Fisica Nucleare, Complesso Universitario di Monte Sant'Angelo, Via Cintia - I-80126 Napoli, Italy \\ ${ }^{2}$ Dipartimento di Fisica, Università di Napoli Federico II, Complesso Universitario di Monte Sant'Angelo, Via Cintia - I-80126 \\ Napoli, Italy
}

\begin{abstract}
We investigate the pairing properties of an effective shell-model interaction defined within a model space outside ${ }^{132} \mathrm{Sn}$ and derived by means of perturbation theory from the CD-Bonn free nucleon-nucleon potential. It turns out that the neutron pairing component of the effective interaction is significantly weaker than the proton one, which accounts for the large pairing gap difference observed in the two-valence identical particle nuclei ${ }^{134} \mathrm{Sn}$ and ${ }^{134} \mathrm{Te}$. The role of the contribution arising from one particle-one hole excitations in determining the pairing force is discussed and its microscopic structure is also analyzed in terms of the multipole decomposition.
\end{abstract}

\section{Introduction}

From the very beginning of nuclear structure studies, pairing has proved to be a main key for understanding many features of nuclei as the odd-even staggering in binding energies, the gap in the excitation spectrum of even-even systems, and the compressed spectrum in odd systems. In spite of that, however, its microscopic understanding is still rather poor.

Starting from the bare interaction among nucleons, it is challenging to try to disentangle the two different contributions that may give rise to the pairing force in finite nuclei, namely the contribution due to the direct attraction between nucleons and that induced by excitations of the nuclear medium. Some works have been recently devoted to the former issue with the aim to investigate the role of the various partial waves of the nucleon-nucleon $(N N)$ interaction as well as that of the Coulomb and the 3-body forces [1-4].

As concerns the effects of the nuclear medium, several works have pointed out that a relevant contribution to the pairing force arises from the exchange of vibrations between nucleon pairs moving in time reversal states close to the Fermi surface $[5,6]$. In all these works, however, phenomenological or semi-phenomenological approaches have been used.

We tackle this problem within a completely microscopic approach by studying the pairing properties of a realistic effective shell-model interaction, derived from the free $N N$ potential in the framework of many-body perturbation theory. Actually, we follow the way traced by Bertsch in his pioneering paper [7], where the $G$ matrix elements derived from the Kallio-Kolltveit potential [8] for the two valence particles in ${ }^{18} \mathrm{O}$ and ${ }^{42} \mathrm{Sc}$ were corrected

\footnotetext{
a e-mail: gargano@na.infn.it
}

by including the core-polarization diagram corresponding to the one particle-one hole ( $1 \mathrm{p} 1 \mathrm{~h})$ excitations. The contribution of this diagram, commonly dubbed "bubble", resulted to be as large as $30 \%$ of the first-order interaction. The very relevant role played by such renormalization in determining the pairing component of the realistic effective shell-model interaction was also pointed out in [9] and [10] for $\mathrm{Pb}$ and $\mathrm{Sn}$ isotopes, respectively.

Over the years, significant progress has been made in the derivation of realistic effective shell-model interactions, and calculations using these interactions have been able to provide an accurate description of nuclear structure properties for nuclei in various mass regions, both close and far from the valley of stability. Nevertheless, little attention has been paid to the approach suggested in the Bertsch's paper. We have started to work along these lines in [11]. Here, based on the results of this paper, we try to go a step forward in the analysis of our effective interaction.

The effective interaction within a model space outside ${ }^{132} \mathrm{Sn}$ is derived from the high-precision CD-Bonn free $N N$ potential [12] by means of the $\hat{Q}$-box folded-diagram expansion [13]. In particular, we study the two valence proton and neutron nuclei, ${ }^{134} \mathrm{Sn}$ and ${ }^{134} \mathrm{Te}$, which allow a direct test of the main features of the neutron-neutron and proton-proton interaction in the presence of the same closed core. The interest in these two nuclei lies also in the fact that they exhibit quite different pairing properties. The experimental data have evidenced, in fact, a large difference between the proton and neutron energy gaps, namely while the first $2^{+}$state in ${ }^{134} \mathrm{Te}$ lies at $1.28 \mathrm{MeV}$, the excitation energy of the corresponding state drops to $726 \mathrm{keV}$ in ${ }^{134} \mathrm{Sn}$. These findings, as well as the most recent mass measurement of ${ }^{134} \mathrm{Sn}$ [14], are well reproduced by our calculations, which have led to results in very good agree- 
ment with experiment also for several other nuclei around ${ }^{132} \mathrm{Sn}$ [15-19]. On these grounds, one should expect that our effective interaction possesses good pairing properties. Therefore, it is worth investigating how they are related to the medium renormalizations.

The paper is organized as follows. Next Section outlines the theoretical framework of our calculations. In Sec. 3 the results for ${ }^{134} \mathrm{Sn}$ and ${ }^{134} \mathrm{Te}$ are presented and the pairing properties of the effective two-body interaction are investigated, focusing attention on the core polarization arising from $1 \mathrm{p} 1 \mathrm{~h}$ excitations. A summary of our conclusions is given in the last Section.

\section{Theoretical framework}

In this Section we shall give an outline of the derivation of our shell-model effective interaction. Particular attention will be devoted to the $1 \mathrm{p} 1 \mathrm{~h}$ core polarization, whose contribution to the neutron and proton pairing will be discussed in detail in Sec. 3. The main steps in the construction of the effective interaction are summarized as follows:

1. The initial input is the CD-Bonn $N N$ potential [12].

2. The high-momentum modes of the CD-Bonn potential, which are unsuitable for a perturbative calculation, are integrated out down to a cutoff momentum $\Lambda=2.2 \mathrm{fm}^{-1}$. This leads to a smooth low-momentum potential $V_{\text {low-k }}$ [20], to which the Coulomb force is explicitly added for protons. For the sake of convenience, in the following we shall refer to the complete interaction, $V_{\text {low-k }}$ plus Coulomb force, as $V_{\text {low-k }}$.

3. The model space is chosen by taking ${ }^{132} \mathrm{Sn}$ as closed core and including the five $0 g_{7 / 2} 1 d 2 s 0 h_{11 / 2}$ proton orbitals and the six $0 h_{9 / 2} 1 f 2 p 0 i_{13 / 2}$ neutron orbitals.

4. The effective interaction is derived within this model space by the $\hat{Q}$-box folded-diagram expansion [13], the $\hat{Q}$-box being composed of one- and two-body diagrams through second order in the interaction. In Fig. 1, we show the four two-body diagrams included in the $\hat{Q}$-box, namely the $V_{\text {low-k }}$, the ladder diagram accounting for excluded configurations above the chosen model space, and two core polarization diagrams $V_{1 \mathrm{p} 1 \mathrm{~h}}$ and $V_{2 \mathrm{p} 2 \mathrm{~h}}$, corresponding to $1 \mathrm{p} 1 \mathrm{~h}$ and $2 \mathrm{p} 2 \mathrm{~h}$ excitations. All diagrams of the $\hat{Q}$-box are computed within the harmonic-oscillator basis with $\hbar \omega=7.88 \mathrm{MeV}$, using intermediate states composed of all possible hole states and particle states restricted to the five proton and neutron shells above the Fermi surface. The shell-model effective interaction is finally obtained by summing up the $\hat{Q}$ box folded diagram series by way of the Lee-Suzuki iteration method [21].

The effective interaction obtained within the approach outlined above contains one- and two-body terms. We employ the subtraction procedure of Ref. [22] to retain only the two-body contribution, $V_{\text {eff }}$, while the single-particle energies are taken from experiment. The adopted singleneutron and -proton energies are reported in [19].

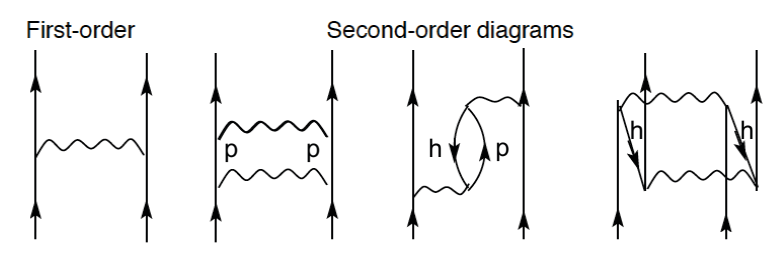

Figure 1. First- and second-order two-body diagrams of the $\hat{Q}$ box

As concerns the class of diagrams corresponding to $1 \mathrm{p} 1 \mathrm{~h}$ excitations, its contribution to a diagonal matrix element is given by

$$
\left\langle j_{a} j_{b}\left|V_{1 \mathrm{p} 1 \mathrm{~h}}\right| j_{a} j_{b}\right\rangle_{J}=\frac{1}{\hat{J}} \sum_{p h J^{\prime}} \frac{1}{\epsilon_{p}-\epsilon_{h}}(-1)^{j_{p}+j_{h}-J^{\prime}}
$$

$$
X\left(\begin{array}{c}
j_{a} j_{b} J \\
j_{a} j_{b} J \\
J^{\prime} J^{\prime} 0
\end{array}\right)\left\langle j_{a} j_{h}\left|V_{\text {low }-\mathrm{k}}\right| j_{a} j_{p}\right\rangle_{J^{\prime}}^{C C}\left\langle j_{p} j_{b}\left|V_{\mathrm{low}-\mathrm{k}}\right| j_{h} j_{b}\right\rangle_{J^{\prime}}^{C C}
$$

the cross-coupled $V_{\text {low-k }}$ matrix elements being defined as

$$
\begin{gathered}
\left\langle j_{a} j_{b}\left|V_{\text {low }-\mathrm{k}}\right| j_{c} j_{d}\right\rangle_{J}^{C C}=\frac{1}{\hat{J}} \sum_{J^{\prime}} \hat{J}^{\prime} X\left(\begin{array}{c}
j_{c} j_{a} J \\
j_{d} j_{b} J \\
J^{\prime} J^{\prime} 0
\end{array}\right) \\
\left\langle j_{a} j_{b}\left|V_{\text {low }-\mathrm{k}}\right| j_{c} j_{d}\right\rangle_{J^{\prime}},
\end{gathered}
$$

with

$$
X\left(\begin{array}{c}
j_{a} j_{b} J \\
j_{c} j_{d} J \\
J^{\prime} J^{\prime} 0
\end{array}\right)=(-1)^{j_{c}+j_{b}+J+J^{\prime}} \hat{J} \hat{J}^{\prime}\left\{\begin{array}{l}
j_{a} j_{b} J \\
j_{d} j_{c} J^{\prime}
\end{array}\right\}
$$

and $\hat{J}=\sqrt{2 J+1}$.

In Eq. (1), the single-particle and single-hole states have the same parity, and the sum runs only over couples of states referring to the same species, namely over $\pi \pi^{-1}$ or $v v^{-1}$ states. This sum is clearly limited by the number of intermediate states we have included in the derivation of $V_{\text {eff }}$.

In order to investigate the effects of this corepolarization contribution on the pairing properties of our effective interaction, it may be useful to analyze its multipole components. This may help understanding the microscopic nature of the low-lying collective surface vibrations, which, as mentioned in the Introduction, have been shown to give rise to a significant induced paring interaction.

By using the multipole decomposition of the $V_{\text {low-k }}$, the $J=0^{+} V_{1 \mathrm{p} 1 \mathrm{~h}}$ diagonal matrix elements can be written as

$$
\left\langle j_{a}^{2}\left|V_{1 \mathrm{p} 1 \mathrm{~h}}\right| j_{a}^{2}\right\rangle_{0}=\frac{1}{\hat{J}_{a}^{2}} \sum_{p h \lambda} \frac{1}{\epsilon_{h}-\epsilon_{p}}(-1)^{\lambda} \hat{\lambda}^{2}\left(w_{\text {ahap }}^{\lambda}\right)^{2},
$$


with the multipole coefficients defined as

$w_{a b c d}^{\lambda}=\sum_{J} \hat{J}^{2}(-1)^{j_{c}+j_{b}-\lambda-J}\left\{\begin{array}{l}j_{a} j_{b} J \\ j_{d} j_{c} \lambda\end{array}\right\}\left\langle j_{a} j_{b}\left|V_{\mathrm{low}-\mathrm{k}}\right| j_{c} j_{d}\right\rangle_{J}$.

From Eq. (2), it may be seen that in this case the $1 \mathrm{p} 1 \mathrm{~h}$ core-polarization contribution takes a quite simple form, as it results from the sum of multipoles, each multipole behaving as a tensor whose rank $\lambda$ is given by the coupling of the particle and hole angular momenta.

\section{$3{ }^{132} \mathrm{Sn}$ region: results and discussion}

Let us start by comparing in the upper and lower part of Fig. 2 the experimental [23] and theoretical spectra of ${ }^{134} \mathrm{Sn}$ and ${ }^{134} \mathrm{Te}$, respectively. The agreement between theory and experiment is very good, and it is worth noting that the very different positions of the $2^{+}$state in ${ }^{134} \mathrm{Sn}$ and ${ }^{134} \mathrm{Te}$ are both remarkably well reproduced. This makes us confident in the reliability of our effective interaction. The accuracy of the $J=0^{+}$two-body matrix elements (TBME) is further confirmed when considering the binding energies, whose calculated values, relative to ${ }^{132} \mathrm{Sn}$, are quite close to the experimental ones. We find $\mathrm{BE}\left({ }^{134} \mathrm{Sn}\right)=6.0$ and $\mathrm{BE}\left({ }^{134} \mathrm{Te}\right)=20.6 \mathrm{MeV}$ to be compared with the experimental value 6.0 [14] and 20.8 [24], respectively.

On these grounds, it may be interesting to analyze the different contributions, i.e. the four diagrams of Fig. 1, entering the construction of $V_{\mathrm{eff}}$ in the attempt to understand their role in determining the pairing force. This study was partially performed in [11], focusing on the nuclear interaction without taking into account the Coulomb force.

In Fig. 3, we report the diagonal matrix elements of $V_{\text {eff }}, V_{\text {low-k }}, V_{\text {lplh }}$, and $\left(V_{\text {low-k }}+V_{1 \mathrm{plh}}\right)$ as a function of $J$ for the $\left(v 1 f_{7 / 2}\right)^{2}$ and $\left(\pi 0 g_{7 / 2}\right)^{2}$ configurations. We choose to devote our attention to these two configurations, since they are the dominant ones in the wave functions of the states shown in Fig. 2 for ${ }^{134} \mathrm{Sn}$ and ${ }^{134} \mathrm{Te}$, the contribution coming from other configurations being, in fact, less than $20 \%$ for each state.

First, we see in both Figs. 3a and $3 b$ that the curves representing $V_{\text {eff }}$ and $\left(V_{\text {low-k }}+V_{\text {lplh }}\right)$ show the same behavior and are quite close to each other. This means that secondorder diagrams other than the $1 \mathrm{p} 1 \mathrm{~h}$ ones and folded diagrams, on the whole, do not give a significant contribution in determining the $v v$ as well as the $\pi \pi$ matrix elements. On the other hand, it is interesting to note that the patterns of the $V_{1 \mathrm{p} 1 \mathrm{~h}}$ curves are completely different in the neutron and proton case. In fact, from Fig. $3 \mathrm{a}$ it appears that the $1 \mathrm{p} 1 \mathrm{~h}$ contribution, being almost flat around $0 \mathrm{MeV}$, affects only slightly the matrix elements of the bare $V_{\text {low-k }}$ interaction. In practice, the matrix elements of $V_{\text {eff }}$ for the $\left(v 1 f_{7 / 2}\right)^{2}$ multiplet are not far from those of $V_{\text {low-k }}$, the largest difference of $250 \mathrm{keV}$ occurring for $J=0$. This means that the effects of the nuclear medium can be neglected in this case.

The $1 \mathrm{p} 1 \mathrm{~h}$ contribution plays a completely different role for the matrix elements of the $\left(\pi 0 g_{7 / 2}\right)^{2}$ configuration as shown in Fig. 3b. In fact, this contribution, starting from a slightly repulsive $V_{\text {low-k }}$ at $J=0$, leads to a large and
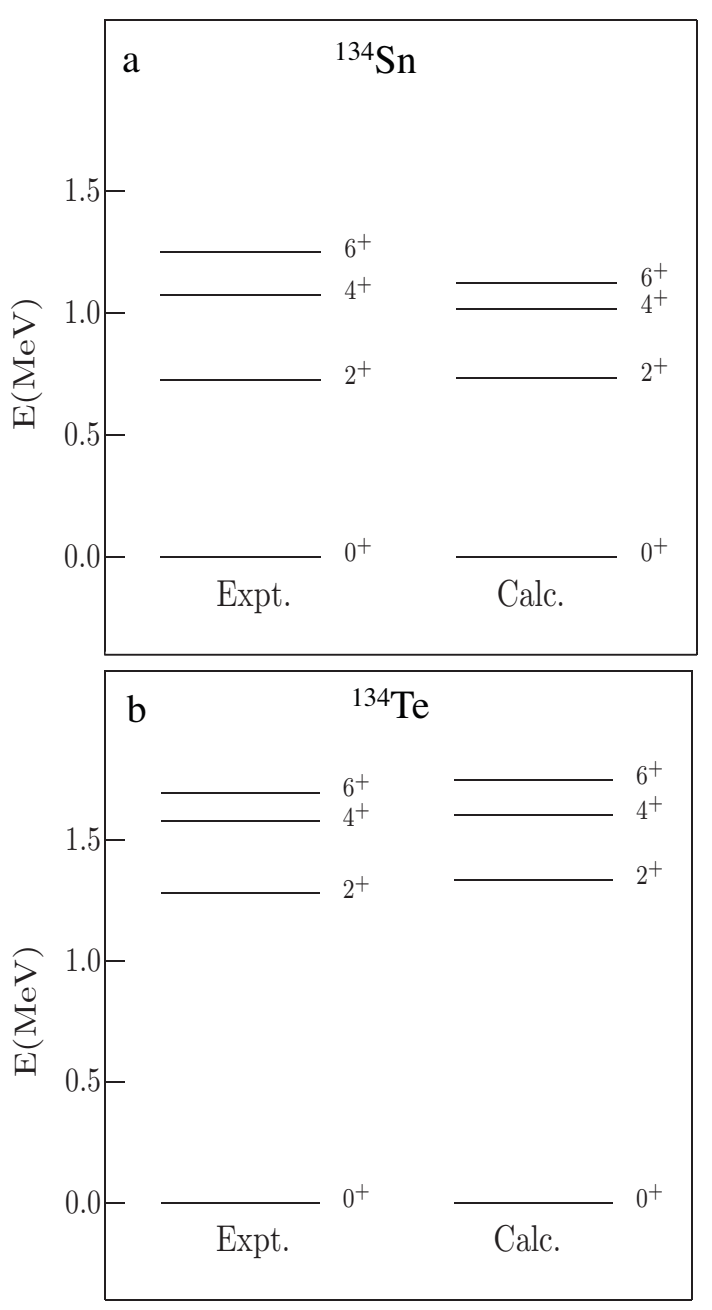

Figure 2. Experimental and calculated spectrum of a) ${ }^{134} \mathrm{Sn}$ and b) ${ }^{134} \mathrm{Te}$

attractive matrix element of $V_{\text {eff }}$, while its effectiveness for higher angular momenta is much less relevant.

The above discussion may be summarized by saying that the sizable pairing force between two protons outside ${ }^{132} \mathrm{Sn}$ may be traced to the large renormalizations induced by the nuclear medium, and, more precisely, by the $1 \mathrm{p} 1 \mathrm{~h}$ excitations, on the bare $V_{\text {low-k }}$ potential. For neutrons, the smallness of these renormalizations gives rise to a weak pairing interaction.

Now, we would like to analyze the structure of $1 \mathrm{p} 1 \mathrm{~h}$ contribution for the $\left\langle\left(v 1 f_{7 / 2}\right)^{2}\left|V_{1 \mathrm{p} 1 \mathrm{~h}}\right|\left(v 1 f_{7 / 2}\right)^{2}\right\rangle_{0}$ and $\left\langle\left(\pi 0 g_{7 / 2}\right)^{2}\left|V_{1 \mathrm{p} 1 \mathrm{~h}}\right|\left(\pi 0 g_{7 / 2}\right)^{2}\right\rangle_{0}$ matrix elements, with the aim to find out if components more relevant than others can be identified. The following discussion does not pretend to be exhaustive, but it may provide the basis for future developments.

In Table'1, we report the values of the two contributions one has when restricting the sum in Eq. (1) to particle-hole proton or neutron states. For the $\left(\pi 0 g_{7 / 2}\right)^{2}$ configuration, the contribution from the $v v^{-1}$ states is large and attractive, while that from $\pi \pi^{-1}$ states is small and repulsive. This simply reflects the major role played by 

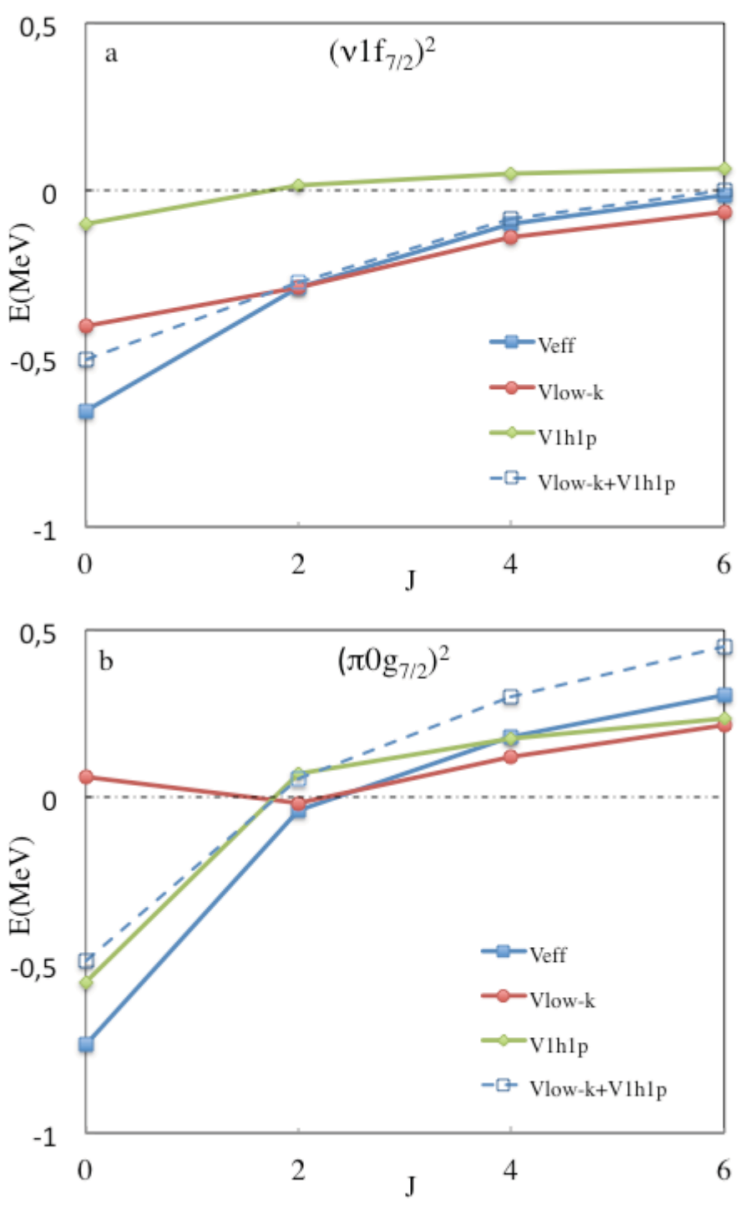

Figure 3. (Color online) Matrix elements of $V_{\text {eff }}, V_{\text {low-k }}, V_{\text {lplh }}$, and $\left(V_{\text {low-k }}+V_{\text {lplh }}\right)$ as a function of $J$ for the a) $\left(v 1 f_{7 / 2}\right)^{2}$ and b) $\left(\pi 0 g_{7 / 2}\right)^{2}$ configuration

neutron-proton $V_{\text {low-k }}$ interaction in determining the $1 \mathrm{p} 1 \mathrm{~h}$ contribution. The same result is obtained for the $\left(v 1 f_{7 / 2}\right)^{2}$, although in this case the contribution arising from the neutron-proton $V_{\text {low-k }}$ interaction is significantly reduced.

Table 1. Contributions from $\pi \pi^{-1}$ and $v v^{-1}$ intermediate states to the $J^{\pi}=0^{+}$diagonal matrix element of $V_{1 \mathrm{plh}}$ for the $\left(v 1 f_{7 / 2}\right)^{2}$ and $\left(\pi 0 g_{7 / 2}\right)^{2}$ configurations

\begin{tabular}{cccc}
\hline$(n l j)^{2}$ & $\pi \pi^{-1}$ & $v v^{-1}$ & $V_{1 \mathrm{p} 1 \mathrm{~h}}$ \\
\hline$\left(v 1 f_{7 / 2}\right)^{2}$ & -0.245 & 0.145 & -0.100 \\
$\left(\pi 0 g_{7 / 2}\right)^{2}$ & 0.021 & -0.570 & -0.549 \\
\hline
\end{tabular}

As next step, we have calculated the multipole contributions entering the two above mentioned matrix elements, namely the various terms appearing in Eq. (2) corresponding to a fixed value of $\lambda$. The even and odd multipole contributions are reported in Figs. $4 \mathrm{a}$ and $4 \mathrm{~b}$, respectively. The behavior of the curves is the same for the $\left(v 1 f_{7 / 2}\right)^{2}$ and $\left(\pi 0 g_{7 / 2}\right)^{2}$ configurations. However, while the odd repulsive multipoles do not give a significant contribution for both configurations, the two curves relative to the even attractive multipoles are largely scaled with respect to each other. As a matter of fact, the contribution relative
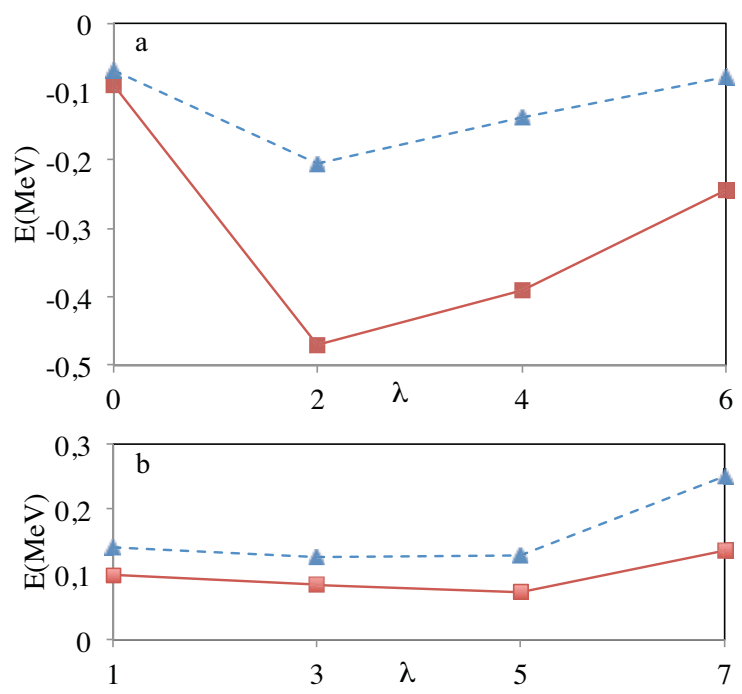

Figure 4. (Color online) a) Even and b) odd multipole contributions to $\left\langle\left(v 1 f_{7 / 2}\right)^{2}\left|V_{1 \mathrm{p} 1 \mathrm{~h}}\right|\left(v 1 f_{7 / 2}\right)^{2}\right\rangle_{0}$ (dashed lines) and $\left\langle\left(\pi 0 g_{7 / 2}\right)^{2}\left|V_{1 \mathrm{p} 1 \mathrm{~h}}\right|\left(\pi 0 g_{7 / 2}\right)^{2}\right\rangle_{0}$ (full lines)

to the $\left(v 1 f_{7 / 2}\right)^{2}$ configuration is rather small, which leads to the scarce relevance of the $1 \mathrm{p} 1 \mathrm{~h}$ renormalizations discussed above. As concerns the $\left(\pi 0 g_{7 / 2}\right)^{2}$ configuration, we see that about $50 \%$ of the renormalization comes from the quadrupole component, but the other even multipoles are also important contributing to the remaining part. This result is in line with the previous calculations by Bertsch for light nuclei [7].

Table 2. $J^{\pi}=0^{+}$diagonal matrix elements of $V_{\text {eff }}, V_{\text {low-k }}$, and $V_{1 \mathrm{plh}}$ for the neutron $(n l j)^{2}$ configurations

\begin{tabular}{lcrr}
\hline$n l j$ & $V_{\text {eff }}$ & $V_{\text {low-k }}$ & \multicolumn{1}{c}{$V_{1 \mathrm{p} 1 \mathrm{~h}}$} \\
\hline $1 f_{7 / 2}$ & -0.654 & -0.403 & -0.100 \\
$2 p_{3 / 2}$ & -0.524 & -0.482 & 0.008 \\
$0 h_{9 / 2}$ & -1.114 & -0.098 & -0.723 \\
$2 p_{1 / 2}$ & -0.179 & 0.003 & -0.104 \\
$1 f_{5 / 2}$ & -0.404 & -0.101 & -0.117 \\
$0 i_{13 / 2}$ & -0.974 & -0.187 & -0.464 \\
\hline
\end{tabular}

Table 3. $J^{\pi}=0^{+}$diagonal matrix elements of $V_{\text {eff }}, V_{\text {low-k }}$, and $V_{1 \mathrm{plh}}$ for the proton $(n l j)^{2}$ configurations

\begin{tabular}{lcrr}
\hline$n l j$ & $V_{\text {eff }}$ & $V_{\text {low-k }}$ & \multicolumn{1}{c}{$V_{1 \mathrm{p} 1 \mathrm{~h}}$} \\
\hline $0 g_{7 / 2}$ & -0.738 & 0.063 & -0.549 \\
$1 d_{5 / 2}$ & -0.486 & -0.304 & -0.157 \\
$1 d_{3 / 2}$ & -0.164 & 0.122 & -0.127 \\
$2 s_{1 / 2}$ & -0.352 & -0.584 & 0.185 \\
$0 h_{11 / 2}$ & -0.912 & -0.080 & -0.757 \\
\hline
\end{tabular}

Finally, it may be interesting to see the effects of the $1 \mathrm{p} 1 \mathrm{~h}$ excitations on $V_{\text {eff }}$ for configurations other than the $\left(v 1 f_{7 / 2}\right)^{2}$ and $\left(\pi 0 g_{7 / 2}\right)^{2}$ ones. To this end, in Tables 2 and 3 we show the $J^{\pi}=0^{+}$diagonal matrix elements of $V_{\text {eff }}$, $V_{\text {low-k }}$, and $V_{\text {lplh }}$ for the two-neutron and -proton configurations. It may be seen that a large $V_{1 \mathrm{plh}}$ contribution can- 
not be considered as something pertaining only to proton orbitals. In fact, sizeable $1 \mathrm{p} 1 \mathrm{~h}$ renormalizations of $V_{\text {low-k }}$ are found for the neutron $\left(0 h_{9 / 2}\right)^{2}$ and $\left(0 i_{13 / 2}\right)^{2}$ configurations, while for protons they play a major role for the $\left(0 h_{11 / 2}\right)^{2}$ configuration besides the $\left(0 g_{9 / 2}\right)^{2}$ which we have already discussed.

\section{Summary}

In this paper, we have discussed the pairing properties of an effective shell-model interaction calculated by means of the $\hat{Q}$-box folded diagram expansion up to second order in the interaction, using a $V_{\text {low-k }}$ derived from the CDBonn $N N$ potential. In particular, we have focused on the matrix elements between two identical particles outside ${ }^{132} \mathrm{Sn}$, starting from a study of ${ }^{134} \mathrm{Sn}$ and ${ }^{134} \mathrm{Te}$, which are characterized by very different paring gaps, the proton gap being $0.5 \mathrm{MeV}$ larger than the neutron one. Based on the very good agreement between theory and experiment, an analysis of the medium effects on the $V_{\text {low-k }}$ has been performed, with particular attention to its $J=0^{+}$matrix elements.

We have found that the many-body effects arising from $1 \mathrm{p} 1 \mathrm{~h}$ excitations play a major role in determining the pairing component of the effective interaction. Actually, the observed difference between the neutron and proton gap is explained as due to the different size in the $1 \mathrm{p} 1 \mathrm{~h}$ contribution to the $V_{\text {low-k }}$ matrix elements for the $\left|\left(v 1 f_{7 / 2}\right)^{2}\right\rangle_{0}$ and $\left|\left(\pi 0 g_{7 / 2}\right)^{2}\right\rangle_{0}$ states, which are the dominant components of the ground states in ${ }^{134} \mathrm{Sn}$ and ${ }^{134} \mathrm{Te}$.

The main points emerged in this work can be summarized as follows; i) the $1 \mathrm{p} 1 \mathrm{~h}$ excitations are very important in determining the pairing component of the nucleonnucleon force in the nuclear medium, providing, in some cases, a contribution larger than that of the bare interaction; ii) the size of the $J=0^{+} V_{1 \mathrm{p} 1 \mathrm{~h}}$ matrix elements depends strongly on the considered configuration and is essentially determined by the neutron-proton interaction and even multipoles, the quadrupole one giving the most relevant contribution.

A preliminary calculation around ${ }^{208} \mathrm{~Pb}$ seems to lead to the same conclusions. However, further work is certainly needed, in particular as concerns the configuration dependence of the $1 \mathrm{p} 1 \mathrm{~h}$ core polarization. This may be helpful to better understand the microscopic structure of low-lying collective surface vibrations whose exchange among $J^{\pi}=0^{+}$pairs of nucleons has been shown to account for a large percentage of the observed paring gaps.

\section{References}

[1] D.J. Dean, M. Hjorth Jensen, Rev. Mod Phys. 75, 607 (2003)
[2] H. Hergert, R. Roth, Phys. Rev. C 80, 024312 (2009)

[3] T. Lesinski, T. Duguet, K. Bennaceur, J. Meyer, Eur. Phys. J. A 40, 121 (2009)

[4] T. Lesinski, K. Hebeler, T. Duguet, A. Schwenk, J. Phys. G: Nucl. Part. Phys. 39, 015108 (2012)

[5] P. Avogadro, F. Barranco, A. Idini, E, Vigezzi, Fifty Years of Nuclear BCS, eds. R.A. Broglia and V. Zelevinsky (Singapore, World Scientific, 2013) pp. 243-262, and references therein.

[6] E.E. Saperstein, M. Baldo, Fifty Years of Nuclear BCS, eds. R. A. Broglia and V. Zelevinsky (Singapore, World Scientific, 2013) pp. 263-273, and references therein.

[7] G.F. Bertsch, Nucl. Phys. A 74, 234 (1965)

[8] A. Kallio, K. Kollveit, Nucl. Phys. A 53, 87 (1964)

[9] G.H. Herling, T.T.S. Kuo, Nucl. Phys. A 181, 113 (1972)

[10] L. Losano, H. Dias, T.T.S. Kuo, Phys. Lett. B 384, 20 (1996)

[11] A. Covello, A. Gargano, T.T.S. Kuo, Fifty Years of Nuclear BCS, eds. R.A. Broglia and V. Zelevinsky (Singapore, World Scientific, 2013) pp. 169-178.

[12] R. Machleidt, Phys. Rev. C 63, 024001 (2001)

[13] L. Coraggio, A. Covello, A. Gargano, N. Itaco, T.T.S. Kuo, Prog. Part. Nucl. Phys. 62, 135 (2009)

[14] M. Dworschak et al., Phys. Rev. Lett. 100, 072501 (2008)

[15] L. Coraggio, A. Covello, A. Gargano, N. Itaco, Phys. Rev. C 80, 021305(R) (2009), and references therein.

[16] L. Coraggio, A. Covello, A. Gargano, N. Itaco, Phys. Rev. C 80, 061303(R) (2009)

[17] A. Covello, L. Coraggio, A. Gargano, N. Itaco, J. Phys. Conf. Ser. 267, 012019 (2011), and references therein.

[18] M. Danchev et al., Phys. Rev. C 84, 061306(R) (2011)

[19] L. Coraggio, A. Covello, A. Gargano, N. Itaco, Phys. Rev. C 87, 034309 (2013)

[20] S. Bogner, T.T.S. Kuo, L. Coraggio, A. Covello, N. Itaco, Phys. Rev. C 65, 051301(R) (2002)

[21] K. Suzuki, S.Y. Lee, Prog. Theor. Phys. 64, 2091 (1980)

[22] J. Shurpin, T.T.S. Kuo, D. Strottman, Nucl. Phys. A 408, 310 (1983)

[23] Data extracted using the NNDC On-line Data Service from the ENSDF database version of September $30,2015$.

[24] J. Hakala et al., Phys. Rev. Lett. 109, 032501 (2012) 